\title{
Significado del verbo PTH en Jeremías 20,7.10
}

El objetivo de este trabajo es poner en claro el sentido de $\mathrm{Jr} 20,7.10 \mathrm{y}$, más concretamente, el significado del verbo PTH, piel y nifal del v. 7 y pual del v. 10. Una traducción como: «Me sedujiste, Yavé, y me dejé seducir», típica y que circula de boca en boca, es, por lo menos, equívoca, debido a la imagen que suscita o/y presupone ${ }^{1}$.

El programa que me propongo desarrollar estudiará: -El sentido resultante de un análisis de todos los textos en que nuestro verbo aparece. - Las consecuencias para el texto en cuestión, lo que supondrá también un análisis de la unidad literaria a la que pertenece.

\section{ANÁLISIS DE LOS TEXTOS}

El verbo PTH es denominativo, procedente de pèt $\hat{\imath}^{2}$. Este sustantivo apa-

1. El lexema «seducir» en castellano significa: «Persuadir al mal con engaños o halagos. // Cautivar el ánimo con algún atractivo físico o moral»: J. CASARES, Diccionario ideológico de la lengua española, Barcelona 1966, p. 759. Se ha polarizado, sin embargo, notablemente, adquiriendo una carga muy fuerte de referencia a la relación hombre-mujer, que aparece connotada en la mayoría de los usos. Ya desde este punto de vista, convendría revisar una traducción semejante. Pero, aun permaneciendo en el sentido original, surge la pregunta sobre si este término no nos pone en una pista equivocada a la hora de interpretar el texto.

2. No existe una opinión unánime sobre si deben considerarse de la misma raíz PTH denominativo de pèt ̂̀ y PTH («ser amplio») procedente del arameo. Para las distintas opiniones, cfr. M. Soebo, pth: E. Jenni - C. Westermann, Theologisches Handwörterbuch zum Alten Testament, München 1971, II, c. 495. Yo sigo esta obra y F. BROWN - S. R. DRIVER - C.A. BRIGGS, Hebrew and English Lexicon of the Old Testament, Oxford 1979, p. 834, considerándolas como dos raíces independientes. Por otra parte, en su segundo significado sólo aparecería con certeza en Gen 9,27 (hifil: «dilatar»). El wahă pittîta de Pr 24,28 se presta a duda, ya que un piel con interrogación parece fuera de toda lógica, dado que el primer estico es una exhortación negativa, si bien el significado de PTH piel sería normal en el contexto (de difamación y engaño). Si, respetando el texto consonántico y cambiando la vocalización en wehiptîta, lo interpretamos como un hifil, la traducción correspondería mejor con la versión de los LXX (medè platýnou), que también está en „ armonía con el primer estico. Tendría el sentido de «dilatarse con los labios». Es precisamente es- 
rece una vez como abstracto $(\operatorname{Pr} 1,22)$ y 18 veces como concreto (14 en $\operatorname{Pr} 3$ en Sal y 1 en Ez). El significado abstracto es «simpleza»; el concreto, «simple», «crédulo». La utilización mayoritaria que de él se hace en los Proverbios nos indica el carácter sapiencial del término. Se dice de una persona que no tiene capacidad de discernimiento, incauta, acrítica y, por lo tanto, fácilmente influible. Esto, en principio, lo sitúa en un campo previo a todo pronunciamiento sobre su cualificación moral. Pero el hecho de aparecer como opuesto a «cauto», «precavido» ('rwm: $\operatorname{Pr} 14,15.18 ; 22,3 ; 27,12$ ), «sabio» (hkm: $\operatorname{Pr}$ 21,11) e «inteligente» ( $n b w n:$ Pr 19,25), es decir, el ideal de hombre en los Proverbios, hace que muy a menudo se utilice despectivamente y se suponga que el pètî es influible para el mal y el error (Ez 45,20; Pr 1,32; 7,7; 14,18; $22,3 ; 27,12)$ y esté apegado a su simpleza (Pr 1,22$)$, siendo, a veces, identificado con «necio» ( $k s y l:$ Pr $1,22.32 ; 8,5 ; 14,18)$. Sin embargo, la razón de la caída es siempre la ignorancia e impercepción, como se dice expresamente en Ez 45,$20 ; \operatorname{Pr} 22,3 ; 27,12$. Por tanto, el concepto de pètî se sitúa en el campo de lo previo: es objeto de la instrucción sapiencial (Sal 19,8; 119,130; $\operatorname{Pr} 1,4 ; 8,5$; 9,14.16), en orden a ser ' $r w m(\operatorname{Pr} 19,25)$ y h hkm $(\operatorname{Pr} 21,11)$. Estos dos últimos conceptos se hallan, pues, en el orden de lo conseguido. Ello aclara cuál es la posición del pètî: puede ser influido para el mal, pero también para el bien. Sólo que, como se trata de una designación sapiencial y los sabios juzgan con el criterio del hombre «conseguido» después de la instrucción, cobra un carácter negativo. Pero la auténtica negatividad se presenta únicamente cuando el pètî se aferra a su simpleza, puesto que tiene como alternativa la posibilidad de aprender. De ahí que en el Sal 116,6 aparezca con una nota de simpatía, como objeto del cuidado compasivo de Yavé.

Tras estas consideraciones, que nos serán de gran utilidad en la evaluación de los datos recogidos, comenzamos el estudio del verbo PTH, distribuido por conjugaciones. Teniendo en cuenta la nota 2 , podemos decir que el número de apariciones es de 26 en total: 4 en qal, 2 en nifal, 17 en piel y 3 en pual. El predominio del piel es evidente.

\section{A. Qal}

1. Job 5,2: wpth tmyt qn'h. Un part. act. en perfecto paralelismo sinoní-

\footnotetext{
te significado el que nos lleva a la otra ocasión en que podría tratarse de un PTH con el mismo significado, «ser amplio», esta vez en qal, también en conexión con «labios»: Pr 20,19. Un participio, con el significado de quien habla demasiado, «el que es amplio de labios». Sea cual fuera la solución al problema de las raíces y aun suponiendo que la opinión que seguimos no sea la última palabra sobre los dos últimos textos, no pienso que represente un impedimento serio para nuestro estudio, pues sólo tres textos - como mucho- entrarían en la segunda categoría. En el trabajo sc prescinde de estos tres textos.
} 
mico con ' $w y l$ del primer estico e insertado en el curso de la primera reconvención de Elifaz. Se trata, por tanto, de una exhortación sapiencial. Habla del destino funesto que espera tanto al pètî como al ' $w y l$ cuando se dejan llevar del enojo y la pasión: «El enojo mata al insensato, la pasión hace morir al simple». El significado, por consiguiente, corresponde prácticamente al concreto de pètî en los proverbios que suponen la caída. Es una comprobación, fruto de la experiencia.

2. Os 7,11: wyhy 'prym kywnh pwth 'yn lb. Part. act. fem., usado en la función nominal de calificativo. Califica a ywnh y va seguido de la expresión privativa 'yn $l b$ («sin cordura»), lo cual nos lleva a $\operatorname{Pr} 7,7$, donde, entre los «simples», se veía un $n^{\prime} r h s r-l b$. El texto se halla en un reproche profético contra las veleidades de Israel, que acude a Egipto y Asiria, siendo devorado por ellos (Os 7,9). El significado continúa siendo el mismo, como lo indica la alusión a la falta de inteligencia, y nada impide que traduzcamos por «simple», «ingenua» o «crédula». Se supone, de hecho, que es presa de todos los engaños.

3. Dt 11,16: hšsmrw lkm pn ypth lbbkm. Un imperfecto, también en conexión con $l b$, pero esta vez con función de sujeto: es el $l b$ quien $y p t h$. Es una advertencia para no caer en el politeísmo cananeo, insertada en una exhortación típica del Deuteronomio. El sentido general es claro, conociendo el significado de pètî y viendo que el versículo continúa con wsrtm. Tiene que ver con el ser engañado y extraviarse. Pero el problema surge cuando, teniendo en cuenta que se trata de un verbo denominativo - como es evidente en los participios-, se intenta dar una traducción. Los diccionarios y muchas versiones traducen por «ser seducido» o «dejarse seducir» ${ }^{3}$. Aparte del problema del «seducir» ${ }^{4}$, nos topamos con otro puramente gramatical: ¿Por qué una traducción pasiva? ¿Cuál es, en ese caso, la diferencia con el nifal y el pual? Mi opinión es que se debería tratar de conservar en la traducción el significado denominativo, aunque se presuponga la caída - lo mismo que con el nombre y los participios-, para respetar la diferencia de conjugaciones. Ahora bien, si el significado de PTH qal es «ser simple», no veo ninguna dificultad en traducir nuestro texto por «cuidad que no sea simple (crédulo) vuestro corazón». Con lo cual nos mantenemos en la misma línea de los textos anteriores.

4.' Job 31,27: wyypt bsstr lbby. Como en Dt 11,16, también aquí un imperfecto (pero invertido) y con $l b$ como sujeto. En este caso se halla en la pró-

3. Nueva Biblia Española: «no os dejéis seducir». Bible de Jérusalem: «Gardez-vous de laisser séduire votre coeur». CEI: «perché il vostro cuore non si lasci sedurre». RSV no traduce exactamente seducir, pero lo pone en pasivo también: «lest your heart be deceived». Lo mismo Brown - Driver - BRIGGS, o. c., p. 834.

4. Cfr. nota 1 . 
tasis de una condicional, en el curso ésta, a su vez, de una serie de condicionales retóricas pronunciadas por Job en su defensa-desafío ante Yavé. Se presentan distintas posibilidades de obrar mal, que merecerían su correspondiente castigo. En este caso se hace mención del culto al sol y a la luna. El problema planteado en la traducción del texto anterior se agudiza ahora con el bsstr, que confiere a la expresión una característica de mayor facticidad. Por lo cual parecería más natural todavía que antes traducir, como lo hacen muchas versiones, por «y fue seducido» 5 . Sin embargo, si queremos ser coherentes con las razones expuestas en el análisis de Dt 11,16, deberíamos intentar la misma traducción: «mi corazón fue crédulo (simple) en lo oculto». Aunque sea, ciertamente, un poco duro al oído, nada nos impide traducir así, lo que parece más en consonancia con el significado de pètî. Debemos, mientras podamos, atenernos al significado original, dejando al contexto la tarea de colorear el término. En este caso es utilizado de forma que presupone el hecho, y no solamente la posibilidad, del ser engañado o seducido. Pero esta misma fluctuación la permite mi propuesta de traducción.

De las cuatro veces que aparece PTH en qal podemos concluir que se mantiene en el mismo campo del sustantivo pètî. Haría falta una mayor frecuencia para una verificación más segura. Pero, en cualquier caso, parece suficiente en principio que estos cuatro textos no impidan la traducción que a priori se ofrece como más lógica. Otro dato material lo constituye el hecho de que las cuatro apariciones en qal responden a la línea negativa de pètî, cosa que no causa extrañeza, debido a la posición que el concepto ocupa en los Proverbios, como ya hemos visto.

\section{B. Nifal}

De las dos veces que aparece el nifal, una es precisamente en Jr 20,7, cuyo significado debe ser matizado en este artículo. Por esta razón me limito ahora al otro caso:

Job 31,9: 'm-npth lbby ' $l$-'šs $h$. El sujeto es de nuevo $l b$. Nos encontramos en la misma apelación defensiva de Job ante Yavé que vimos ya en Job 31,27 y se trata también de la prótasis de una condicional retórica que presenta otra posibilidad de obrar el mal. Va seguido de la preposición ' $l$, que se presta a confusión. Dos preguntas complementarias deben ser respondidas para penetrar en el texto a nivel de estructura gramatical: ¿Cuál es la especificidad del nifal? ¿Cuál es el sentido de la preposición ' $l$ ?

Traducciones como «si mi corazón fue seducido por mujer» y similares ${ }^{6}$,

5. Así traducen todas las versiones citadas en la nota 3 .

6. Traducen de esto modo: Nueva Biblia Española, Bible de Jérusalem y CEI. RSV conser- 
aunque respondan al sentido del texto, inducen a error. Aun suponiendo que el nifal tenga un significado pasivo en nuestro texto, no se puede poner «mujer» como ablativo agente. El pasivo hebreo no lo tiene, en general, y las pocas veces que aparece atestiguado lo hace con las preposiciones $m n, b$ y $l$, nunca con $\zeta\urcorner$. $l$, en cambio, es empleada en algunos textos como sinónimo de $l$, expresando la dirección del corazón y la mente $(l b)^{8}$. Si nuestro versículo fuese uno de esos casos, el movimiento de la acción iría del $l b$ de Job a ’̌v̌̌ $h$. Que la cuestión se pueda resolver así o no, depende, en última instancia, de las posibilidades que el nifal ofrezca.

Entre los diversos sentidos teóricos que el nifal puede tener, hay que encontrar uno que respete lo dicho en el párrafo anterior, es decir, el movimiento de la acción $l b$ - ' $̌ s ̌ h$. Se deben descartar, por un lado, el reflexivo y el tolerativo (eficaz), que suponen una acción transitiva en el qal ${ }^{9}$. Por otra parte, el simplemente pasivo no cuadra con la preposición $\%$. Por lo cual, parece más razonable optar por un sentido resultativo, aunque, dado el significado del qal, tiene mucho de pasivo. Acentuaría el estado adquirido tras un proceso. Con ello, el sentido negativo del «ser simple» se afirma mucho más rotundamente. La traducción podría ser algo así como «si mi corazón se atolondró por una mujer», donde «se» equivale al final de un proceso («haberse atolondrado») y «por» indica tanto la dirección del sentimiento como la causa.

\section{Piel-pual}

Ya de entrada se nos plantea la pregunta de cuál puede ser la peculiaridad semántica del piel con relación al qal y al sustantivo pètî. Puesto que, como veremos, designa una acción transitiva, será cuestión de examinar sujeto y complemento directo, así como la descripción de la acción misma.

\section{Sujeto, un hombre}

\section{1,1. Jc 14,15: ptty 't-'yšk wyggd-lnw 't-hh̆ydh} Jc 16,5: ptty' 'wtw wr'y...

Son dos textos en los que a Sansón le sonsacan, en el primero su mujer y en el segundo Dalila, un secreto que no quería revelar a nadie. En ambos casos a las mujeres les es requerida la acción bajo amenazas por parte de los filis-

\footnotetext{
va mejor el sentido de la preposición ' $l$ : «If my heart has been enticed to a woman». También Brown - Driver - Briggs, o. c., p. 834: «enticed unto...».

7. Cfr. P. Jouon, Grammaire de l'hébreu biblique, Rome 1923, $132 \mathrm{c}$.

8. Cfr. Brown - Driver - Briggs, o. c., p. 757.

9. Cfr. P. JoUON, o. c., $51 \mathrm{c}$.
} 
teos. De ahí que se trata en lós dos de un imperativo femenino. En el c. directo se alude siempre a Sansón ' $y s ̌ k$, ' $w t w$. En 14,15 la expresión va seguida de un $w^{\text {e } y i q t o l ~ d e ~ f i n a l i d a d, ~} 16,5$ de otro imperativo que explicita el fin perseguido con el ptty. El tema es la manipulación que sufre el protagonista del relato con vistas a comunicar un secreto, lo cual le acarreará un fuerte perjuicio económico en el primer caso y la cautividad con la muerte en el segundo. Aunque la acción es promovida por los filisteos, el agente del PTH son las mujeres. Los relatos nos dicen, además, en qué consiste este PTH. Las mujeres desarrollan un acoso pertinaz, juegan con la paciencia y los sentimientos de Sansón, hasta que él, importunado y aburrido, se rinde.

En estos primeros textos analizados del piel podemos observar ya la característica que se repetirá en todos los demás, con relación al sustantivo pètî. Si alquien representa al pètî de algún modo, ése es Sansón, el cual es manipulado y sacado de su entereza mediante una «técnica» que lleva consigo segundas intenciones y que triunfa sobre él. Universalizando: el c. directo representa al pètî. Verificaremos esta afirmación en cada uno de los textos analizados.

Al tratar de traducir nos topamos con una dificultad, que, a medida que avancemos en el estudio, se convertirá en una segunda característica: la necesidad de recurrir a la idea contenida en el sustantivo pèt $\hat{\imath}$, por un lado, y, por otro, concretar según el contexto. En Jc 14,15; 16,5 esta concretización nos la ofrece la descripción de la escena que las mujeres montan para debilitar la resistencia de Sansón. Ésta es la auténtica explicación del ptty, ya que sujeto y c. directo sólo nos informan de los personajes. Según esto, la traducción dependerá, en cierto modo, de lo genérica que se quiera dejar, dentro de la misma concreción del texto. En este caso la traducción puede oscilar entre un «engañar-manipular» y un más concreto «persuadir» o «sonsacar».

1,2. 2S 3,25: ky lptttk b' wld't... Tenemos un infinitivo constructo con $l$ de finalidad. Representa, según la opinión de Joab, la razón por la que Abner ha venido a ver a David. No tenemos en esta ocasión una descripción de la actividad de Abner que nos ayude a descifrar el sentido, pero este primer infinitivo es puesto en paralelo con los dos wld't que siguen en la segunda parte del versículo. Estos dos infinitivos deben ser interpretados, pues, como la explicación de nuestro verbo. Abner ha venido para enterarse de todos los proyectos y movimientos de David. El contexto nos aclara que estamos ante una actividad de espionaje. En tal contexto, la raíz que estudiamos se presenta como lógicamente utilizada. Se trata de un trabajo de astucia: entrar en el campo enemigo y granjearse la amistad del jefe para enterarse de lo que quiere. El hecho de que sea sólo la versión ficticia de Joab y no la realidad sobre Abner no cambia en nada la lógica de la argumentación. La traducción, por tanto, sería prácticamente la misma que en los dos textos anteriores. Sería preferible man- 
tenerse en un genérico «engañarte», puesto que no disponemos de una descripción de la acción.

1,3. $\operatorname{Pr} 25,15:$ b'rk 'ppym yptth qșyn. Es ahora un pual y, por consiguiente, será el sujeto el que haga las veces del pètî, pues corresponde al c. directo en el piel. Estamos ante un hecho de experiencia codificado en un proverbio: el autodominio y la paciencia constituyen una inmensa fuerza. En qué consiste el yptth, no es aclarado. Remitiéndonos al significado general de la raíz, podemos decir que se trata de hacer cambiar algo en la mente del qșn y la misma paciencia constituye la superioridad estratégica que lleva al triunfo. «Convencer» o «persuadir» se presentan como traducciones lógicas en un contexto que parece oponer dos voluntades. Se pone el acento en un procedimiento de astucia, que no es descrito ulteriormente. Con ello se va aclarando también que nuestro término tiene que ver más con la oposición incautoastuto que bueno-malo en nuestra concepción moral. Así lo demuestra el hecho de que la «moralidad» se ponga entre paréntesis en textos como el que nos ocupa. Con otras palabras, se trata de un concepto sapiencial y de una ética sapiencial, como se dijo al principio al hablar de pètî. El modelo es el hombre paciente y frío, que sabe desenvolverse en todas las circunstancias y cambiar una situación comprometida en favorable.

1,4. Sal 78,36: wypttwhw bpyhm wblšwn ykzzbw-lw. Imperfecto invertido con sufijo (c. directo) de $3 .^{\mathrm{a}}$ sing. El sufijo se refiere a Dios. Hay un paralelismo sinonímico y una composición quiástica de los esticos. bpyhm y wblswnm son equivalentes, ambos son órganos de la palabra. wypttwhw y $y k z z b w-l w$ deben ser tomados como conceptos próximos. Hay que proponer como término básico un «engañaban», que, por razón del contexto (conversión insincera), puede colorearse con un «adulaban» o «halagaban» más concretos (con el fin de granjearse el perdón de Dios, que, a su vez, debía mejorar la situación desastrosa). Es obvio que en este caso tiene fuertes connotaciones negativas, puesto que el objetivo es un manipular a Dios para que actúe al antojo del hombre, es dejarlo en la posición de un «simple» (c. directo).

1,5. $\operatorname{Pr} 1$ 1,10: bny 'm-ypttwk htt'ym 'l-tb'. Imperpecto con sufijo (c. directo) de $2 .^{a}$ sing. Es una exhortación sapiencial (bny) a resistir àl ypttwk de los pecadores. Por los versículos que siguen se puede ver qué es lo que persiguen los pecadores: atraer a su conducta de violencia. Esto nos permite traducir por «seducir», tomado en su significado original, o, más neutralmente, por «extraviar». Sólo que, dado que la acción no aparece como alcanzando su objetivo, puesto que la resistencia para neutralizarla es posible ('l-t $t$ '), una traducción correcta impone un modo de especificar la acción en curso, lo que se consigue con un verbo de conato: «Si intentan extraviarte (seducirte) los peca- 
dores». Se podría decir que Pr 1,10 está en la línea de los textos estudiados al principio en los que el pètî era objeto de la instrucción sapiencial. Detrás del bny se esconde el mismo tipo de persona. Allí se hablaba a un sujeto que tenía que salir de una situación de ignorancia perniciosa, aquí se exhorta al discípulo a superar la acción perversiva a la que un pètî puede y suele sucumbir. Es interesante comprobar que la misma raíz es utilizada tanto en una como en otra dirección mediante el juego de las conjugaciones.

1,6. $\operatorname{Pr} 16,26:$ 'yš ḥms yptth r'hw whwlykw bdrk lw'-twb. Otro imperfecto. Es muy semejante al caso anterior en cuanto a la semántica, si bien se trata ahora de una constatación ( $3 .^{a}$ persona) sin tono exhortativo explícito. El paralelismo, unido a la secuencia verbal (yiqtol-we qataltî), aconseja ver en el segundo estico la acción subsiguiente al yptth «conseguido» ( $w^{\mathrm{e}}$ qataltî de consecución): «lo lleva por un camino no bueno».

1,7. Ex 22,15: wky-yptth 'yš btwlh 'šr lw'-'rs̀h wškb 'mmh. Imperfecto en un texto legal. El c. directo es una muchacha soltera. Lo mismo que en el texto anterior, la secuencia verbal (yiqtol-we qataltî) da idea de consecución y, por tanto, explica una acción ulterior al yptth, que lo completa y realiza. Se trata ahora de la relación sexual. La traducción más adecuada es: «Si un hombre seduce a una joven...». Seducir se debe tomar aquí con el mismo sentido que en las novelas y el teatro predomina en nuestras lenguas, en el ámbito de las relaciones hombre-mujer ${ }^{10}$.

\section{Sujeto, Dios o representante de Dios}

2,1. Os 2,16: lkn hnnh 'nky mpttyh whwlktyh hmmdbr wdbbrty 'l-lbbh. Part. masc. con sufijo de 3 . $^{\text {a }}$ fem. sing. Como en los dos casos anteriores, los dos $w^{e}$ qataltî que siguen al participio expresan sucesión, por lo menos, y, como se trata de acciones que se producen gracias a la primera, también consecución. Yavé se presenta a sí mismo como quien mptth. El pronombre sustituye a Israel, esposa infiel. El contexto (Os 2,4-25) nos presenta a Yavé lamentándose por la infidelidad de Israel, que se ha convertido en una prostituta. El proyecto de Yavé es atraerla de nuevo. En la lucha con sus rivales, Yavé debe utilizar un arte de captación que cambie la situación de fornicación en fidelidad. Este contexto aconseja traducir por «atraer» o «seducir». Pero conviene notar que hay un doble juego. El concepto «seducir», en castellano, implica siempre una connotación moral negativa y sólo en un contexto literario lírico, lleno de contrastes y juegos de sentimientos, como es el que nos ocupa, ad-

10. Cfr. nota 1 . 
quiere un matiz positivo. En hebreo, sin embargo, no parece que esto sea tan claro. Si, como va manifestándose por ahora, se trata de un concepto en el que predomina el factor astucia, siendo secundarias la bondad y la maldad, sería utilizado aquí con toda propiedad, sin ningún salto, como una de las posibilidades concretas del verbo.

2,2. $1 R$ 22,20.21.22 // 2Cró 18,19.20.21: Es el relato del profeta Miqueas ben Yimlá ante Ajab. Miqueas cuenta al rey la visión que ha tenido. En ella se presenta a Yavé con todo el ejército celestial pidiendo un voluntario: $m y$ yptth 't ' $h$ ' $b$. Un espíritu se ofrece: 'ny 'pttnnw. Y la pregunta de Yavé de cómo lo hará, responde: 'ṣ' whyyty rwh šq $q$ r bpy kl-nby'w. Yavé da su aprobación, afirmando el éxito por adelantado: tptth wgm-twkl. El sujeto de PTH piel es, en los tres casos, un espíritu enviado por Yavé. El c. directo es siempre Ajab. El fin, hacer subir a Ajab a Ramot de Galaad para que muera. El medio (la concreción del PTH) son los falsos profetas y sus profecías engañosas. Vemos, por tanto, que el yptth es realizado mediante un $r w h \grave{s} \stackrel{v}{s} r$, que aparece de nuevo en el versículo siguiente. Este dato nos permite traducir con un genérico «engañar», aunque habría que suponerlo con las características de un más concreto «manipular». Aparece de nuevo el aspecto de superioridad estratégica y de astucia, cuyo usufructuario en esta ocasión es Dios.

2,3. Ez 14,9: whnnby' ky-yptth wdbbr dbr 'ny yhwh pttyty 't hnnby' $h h w$. Es una condicional, en cuya prótasis aparece nuestro verbo en pual, mientras que en la apódosis tenemos un piel. El personaje que sufre la acción es el profeta en ambos casos (sujeto del pual y c. directo del piel). En cuanto a quien la realiza, es claramente Yavé en la apódosis (sujeto del piel), pero no parece serlo en la prótasis. Es curiosa la secuencia imperfecto-perfecto, que, $\sin w$ conversivo, remite la apódosis al pasado, estando la prótasis en un presente de casuística legal. El texto habla de una consulta a Yavé mediante el profeta, que no debe ser hecha, y, en caso de hacerse, no debe ser respondida. La condicional supone la eventualidad de que el profeta sucumba a las instancias de quien viene a consultar. Según esto, el sujeto de la prótasis sería precisamente la persona que consulta al profeta. La apódosis implicaría, por consiguiente, que Yavé reivindica para sí la acción del PTH, que es él mismo quien yptth al profeta. El perfecto se podría interpretar, en tal caso, como un futuro perfecto: «habré sido yo quien ha...» ${ }^{11}$. La situación nos recuerda el texto anterior, en el que Dios se valía de la misma mentira de los profetas de Ajab para perderlo. Aquí utiliza igualmente los medios que ofrece la situación de corrup-

11. Cfr. P. Jouon, o. c., $112 \mathrm{i}$. 
ción idolátrica. Si en 1R // 2Cro se decía que el espíritu enviado por Dios se había hecho $r w h$ š qr en los profetas de Ajab, en Ez 14,19 se afirma que Dios asume las triquiñuelas de quien viene a consultar. Son dos modos de expresar la misma idea nuclear -en lo que importa para nuestro trabajo-: Dios se sirve de la situación engañosa general para engañar a un personaje central de ella. Así que la traducción de Ez 14,19 debería ser equivalente a la de los textos de $1 \mathrm{R}$ y 2 Cro.

Es de notar que, aunque se haya hecho una división metodológica entre los textos en que el sujeto del verbo PTH piel es un hombre y aquellos en los que esta función la ejerce Dios, no se halla ningún rasgo cualitativamente distinto en estos últimos. El verbo es usado siempre al mismo nivel semántico. Se habla de una acción de Dios, el cual pone en marcha ciertos mecanismos para cumplir sus planes, exactamente igual que en la primera serie de textos.

\section{Conclusiones sobre el piel-pual}

Una primera observación es la imposibilidad de traducir siempre con la misma palabra en castellano, como ya ocurre con el griego en los LXX. Esto puede ser debido a dos causas: o que se trata de un lexema genérico que cubre la acción descrita por distintos lexemas en nuestra lengua (los propuestos en las traducciones), o que es más bien un término específico no existente en las lenguas indoeuropeas y que cubre una zona de intersección de la acción descrita por los distintos términos con que hemos traducido.

Para resolver la disyuntiva debemos recurrir, una vez más, al origen del verbo, como ya apunté al principio, o sea, al sustantivo pètî, de carácter sapiencial. Si, como se afirmaba entonces, el c. directo del piel y el sujeto del pual representan el papel del pètî, quiere decir que sufren una de las acciones a las que el pètî se hace normalmente acreedor. O lo que es lo mismo, el PTH piel es un lexema específico que designa el tipo de acción que se hace con un pètî en su condición de tal. Sujeto y c. directo se hallan en la misma relación que 'rwm-pètî. Todos los textos estudiados parecen confirmar esta hipótesis.

De ahí viene la imposibilidad de encontrar una traducción completamente adecuada y mis titubeos en los párrafos que preceden. Todos y cada uno de los lexemas usados son válidos sólo ocasionalmente, en el texto concreto que traducen, pero contienen otra serie de semas que no corresponden al significado específico de PTH. Dos son, pues, los problemas: el significado del verbo hebreo y la traducción del texto. La traducción de un solo texto no puede ofrecernos el auténtico sentido del verbo hebrero.

Los conceptos paralelos con los que aparece nuestro verbo deben ser considerados como el despliegue de las posibilidades que contiene, en una perfecta correspondencia con lo visto para el sustantivo pètî. PTH piel aparece tam- 
bién como un influjo - basado en la astucia, superioridad en experiencia, inteligencia, etc. - en orden a dejar al otro a merced de quien lo ejerce. Puede ser para el mal (la mayoría de los casos), pero también para el bien (Os 2,16), o con una finalidad de provecho para el autor de la acción y neutra para el que la sufre $(\operatorname{Pr} 25,15)$. Es decir, el acento se pone en la superioridad de astucia; la bondad-maldad y el perjuicio-beneficio están en un plano secundario, como consecuencias implicadas en esta superioridad.

Una verificación la tenemos en la diversidad de contextos y géneros literarios en que aparece el lexema. La temática no coincide exactamente en ninguno de los textos y depende siempre de la situación y condición del personaje que sufre la acción. Los géneros literarios van deśde la narrativa histórica al lenguaje legal, pasando por proverbios, un salmo y oráculos proféticos. Prueba ésta evidente de que lo que importa es la idea central antes explicada, que, por lo demás, es aplicable a un sinfín de situaciones distintas con esa característica común.

II. $\operatorname{Jr} 20,7.10$

\section{A. Unidad literaria a la que pertenece}

Me limitaré a poner de relieve los datos que estructuran la unidad y en torno a los cuales se desarrolla la acción. No nos interesa directamente precisar con detalle el tipo de composición de que se trata, sino únicamente ver el talante del texto. Un estudio exhaustivo sería iluminador, por supuesto, pero alargaría demasiado el trabajo.

\section{Vocabulario}

Predominan los lexemas de fuerza, violencia, oposición, y los medios utilizados (burla, acechanzas). Destaca, en este sentido, la repetición del verbo $y k l$ solo, sin acompañar a otro verbo (vv. 7.9.10.11), con el significado de prevalecer sobre alguien o algo. Asimismo nqmtnw (v. 10) - nqmtk (v. 12). Interesante es el hecho de que el verbo PTH se repita acompañado de $y k l$ en los vv. 7 (piel-nifal) y 10 (pual). La agresión de los opositores aparece en los vv. 7-8 bajo la forma de $l s h w q, l w ' g, h r p h$, $q l s$, términos todos relativos a la irrisión, el ridículo y la befa; en el v. 10 se describe en términos de acecho ( $s w m r y s ̦ l^{\prime} y$, 'wly yptth). A lo cual correspone la venganza de Yavé en términos de «confusión eterna» (klmmt 'wlm) (v. 11). Es también un dato importante, y no tạ evidente, que la expresión «como fuego ardiente» ( $\left.k^{\prime} s w^{\prime} b t\right)$, del v. 9, pertenece igualmente al ámbito semántico de la violencia y de ningún modo debe ser considerada como una descripción del celo de Jeremías o cualquier otro tipo de afecto similar. Lo prueba la lectura de los textos en que las dos raíces aparecen juntas: se refiere directamente a la ira de Yavé en Nm 11, 1-3; Is 
30,$27 ; \mathrm{Jr} 4,4 ; 21,12 ;$ Sal 79,5; 89,47; Lm 2,3; y a la violencia de Yavé en $2 \mathrm{~S}$ $22,9.13 ; \mathrm{Jb} 1,16$; es el elemento que circunda la voz de Yavé y causa temor en Dt 4,11; 5,23; $(9,15)$; es maldad que devora en Is 9,17; destrucción en Sal 83,$15 ; 106,18$; y estado de uno que se lamenta en Sal 39,4. Según esto, en nuestro contexto lo más verosímil es que se trate de una agresión de Yavé, como se verá en el cuadro de relaciones.

\section{Esquema de relaciones}

Explicación de los signos del cuadro de la página siguiente:

(1) Relación de benignidad y ayuda

(2) Relación de hostilidad sin especificar el éxito

(3) Relación de hostilidad triunfante

(4) Relación de hostilidad fracasada

El verbo $y k l$ ejerce una función especialmente estructurante, ya que aparece en la acción de los tres personajes.

Como puede verse en el cuadro, se produce una tensión en los vv. 7-10, que es resuelta en 11-12. Yavé pttytny y ha prevalecido ( $t w k l)$, lo mismo que se dice del intento de los opositores en 10bA. Del mismo modo, Jeremías es impotente frente a Yavé ( $l w^{\prime}$ ' $w k l$ : $\left.9 \mathrm{~b}\right)$. La palabra de Yavé se ha tornado para Jeremías oprobio y burla (v. 8), porque le ha granjeado el odio y la oposición de la gente $(7 \mathrm{bB})$, hasta el punto de que la agresión de Yavé y lạ de los opositores son casi identificadas $(7 \mathrm{a}=10 \mathrm{bA} .7 \mathrm{~b})$. Jeremías se ve atacado por dos frentes. La tensión llega a su clímax en 10bA, como hemos visto. Hay, sin embargo, una diferencia entre 10bA y 7a: la oposición de Yavé aparece como rotunda y con éxito completo (nifal en $1 .^{a}$ persona de $7 \mathrm{aA}$, wayyiqtol de $7 \mathrm{aB}$, negación de la propia fuerza en $9 \mathrm{bD}$ ), mientras que la de los enemigos es consistente, pero no triunfante, en conato (10aCD.b), y apoyada en la eventual identificación o complementación con la oposición de Yavé (7b.8b).

Es éste desequilibrio de fuerzas el que resuelve la tensión, sobre todo si se tiene en cuenta que la oposición de la gente ha sido provocada por el triunfo de Yavé ( $k y$ causal de 8a), que hace gritar a Jeremías cosas infaustas. Yavé se pone definitivamente del lado de Jeremías. El enemigo triunfador se convierte en aliado triunfador contra los enemigos más flojos. Yavé siempre prevalece, mientras los opositores no (11aB.bA). A la venganza proyectada de los opositores $(10 \mathrm{bB})$ se opone la venganza realizada de Yavé. La derrota de los enemigos será definitiva (12bB).

\section{Determinación de la unidad literaria}

No hay duda de que al menos los vv. 7-12 pertenecen a la misma unidad, 
$\begin{array}{ll}\text { v. } 7 & \text { pttytny w'ppt } \\ \text { hazzqtny wttwkl }\end{array}$

v. 8

$$
\text { 'z'q }
$$
kllh lw'g ly

. hms wš̀ 'qr

v.9 dbr-yhwh ly

(2)

(2) lw'-'zkrnnw

(2) lw'-'dbbr bšmw

k's bw'rt

(2)

nl'yty klkl

(4) lw' 'wkl

v. 10

(2) dbbt rbbym

(2) (2) -............. hggydw wnggydnnw

(2)

(2) šwmry șl'y

(2) 'wly yptth

(2) wnwklh lw
(4) ykkšlw

(4) lhrph wlqls

v.11 yhwh 'wty kgbbwr 'ryṣ

(4) lw' yklw

v. 12 bwḥn șddyq (1) lw' hs̀kylw nqmtk mhm

v. 13

(1)

'lyk gllyty 't-ryby

(1) syrw

(1) hllw

hṣyl

myyd mr'ym 
después de ver el entramado de relaciones y el progreso de la acción, así como las repeticiones de términos. Digo «al menos», pues se plantea la pregunta sobre el v. 13, en el que no hemos visto ningún elemento de los comunes a 7-12, pero que, desde luego, no encaja en 14-18. Estos últimos versos constituyen una automaldición, organizada en torno al vocablo 'rwr y cerrada en sí misma. No se insertan en el curso de la acción de 7-12, que es lógica y acabada. El v. 13, sin entrar ahora en problemas de redacción, se sitúa más lógicamente como conclusión de 7-12 ${ }^{12}$. Se trata de una invitación a la alabanza de Yavé, que viene tras la declaración de confianza.

En 7-13 tenemos, en vista de lo dicho, una oración, cuyo esquema se parece bastante - por lo menos - al de un salmo de lamentación. La estructura de esta unidad podría ser:

7a. Victoria de Yavé sobre Jeremías

7b-8. Consecuencias: burla de la gente contra Jeremías

Lamentación 9. Oposición inútil de Jeremías

10. Acechanzas de los opositores

11aA. Yavé se alía con Jeremías

Declaración 11aB-12. Consecuencias: la derrota de los enemigos será definitiva de confianza

13. Invitación a la alabanza.

\section{Conclusiones}

Se podría objetar que la mención de la palabra de Yavé y de la actividad proféticà en el v. 8, así como la alusión a hablar en el nombre de Yavé y acordarse de Él en el v. 9, nos llevan a una situación de fidelidad a Yavé y no de lucha. No se puede negar el dato y quizá habría que verlo en el contexto de las otras «Confesiones de Jeremías». Pero, en todo caso sa fidelidad en nuestro texto es presentada conscientemente como forzada, y esto es lo que nos importa. No se habla de una respuesta voluntaria, sino más bien de una fuerza que hace inútil cualquier oposición - la oposición de Jeremías es, a todas luces, clara- Como mucho, se puede hablar de una fidelidad aregañadientes, malgré lui. Por otra parte, el texto no habla de una primera llamada a la vocación, sino de una situación de desamparo en que las desgracias y la vocación se hallan en conexión íntima. De ahí la imagen resultante y el predominio de las relaciones de lucha, aunque éstas sean un elemento retórico de la oración. Es

12. Para un tratamiento detallado, cfr. D.J.A. CLINES - D. M. GUNN, «Form, Occasion and Redaction in Jeremiah 20»: $Z A W, 88$ (1976) 390-409. 
significativo, a este respecto, que en la declaración de confianza la respuesta de Yavé sea de violencia contra los enemigos. Ésta es el signo de que Yavé se ha puesto de parte de Jeremías. No queda terreno para la neutralidad. Lo cual es una razón más para concluir que la primera parte está igualmente compuesta en clave de violencia y no representa ninguna relación idílica entre Jeremías y Yavé.

\section{B. Sentido del verbo PTH}

En la primera parte del artículo se ha esclarecido que el significado de PTH piel era, específicamente, «lo que se hace con un simple» y que la coloración situacional le viene del contexto. En II A se ha definido el contenido de Jr 20, 7-13 como uno en el que prevalecen las relaciones de violencia y oposición. En línea con estas conclusiones propondré mi interpretación del verbo en cuestión.

\section{1. $E l v .10$}

Este verso es fundamental para el discernimiento del sentido. yptth se encuentra allí en paralelismo con $\stackrel{s}{w} w r y s l^{\prime} y$. El resultado de ello, perseguido por los opositores, es wnwklh lw y wnqhh nqmtnw mmmnnw ( $w$ más cohortativo de finalidad). El sentido sería, teniendo en cuenta que es pual, «caer en el engaño (trampa, maniobra, manipulación, acechanza...)». Los opositores esperan un momento de flaqueza, inadvertencia, un «traspié», para «prevalecer» por medio de su estrategia.

2. $E l v .7$

En el v. 7 hay que suponer, debido al paralelismo, un significado similar ${ }^{13}$. 7a está dividido en dos expresiones paralelas:
7aA: pttytny yhwh w'ppt
7aB. haqtny perfecto wayyiqtol wttwkl perfecto wayyiqtol

Si el wayyiqtol indica normalmente sucesión y, en algunos casos, como podría ser éste, consecución (dos acciones del mismo sujeto que se siguen temporalmente, dependiendo la segunda de la primera), podemns traducir $7 \mathrm{aB}$ : «fuiste

13. Además del fenómeno del paralelismo, está el hecho de que en los vv. 7 y 10 se dan las tres únicas apariciones del verbo PTH que existen en el libro de Jr. Es una fuerte razón para suponer que, supuesta igualmente la unidad redaccional, sean utilizadas con el mismo sentido. 
más fuerte que yo (me dominaste), y (de forma que) has prevalecido». Dos cosas importantes: -la directa connotación de dominio mediante la fuerza; -la secuencia narrativa.

$\mathrm{Si}$, como se ha mostrado en la estructura, 7a declara la victoria de Yavé sobre Jeremías, esta secuencia (perfecto-wayyuqtol) nos indica que la victoria (waayyiqtol) es la consecuencia de una superioridad (perfecto) neta de Yavé.

Mientras que en $7 \mathrm{aB}$ la superioridad es de fuerza, en 7aA la superioridad es en lo peculiar del verbo PTH piel: la «estrategia». Yavé es más fuerte y, además, ha dispuesto la lucha de forma que ha sorprendido en su simpleza a Jeremías. En la secuencia verbal de 7aA, pttytny representa la acción conativa de Yavé y w'ppt la consecución del fin, el éxito de la empresa. La traducción que propongo, para respetar el paralelismo con el v. $10 \mathrm{y}$ lo dicho sobre el nifal, es ésta: «Me indujiste a engaño, Yavé, y (de forma que) yo me atolondré (quedé atolondrado). Fuiste más fuerte que yo, y (de forma que) has prevalecido». El nifal encaja con toda normalidad en el significado resultativopasivo. El pual, como pasivo del piel, se refiere a la acción transitiva en cuanto tal, que puede ser conseguida o no.

Un respaldo para la traducción dada nos lo ofrece $1 \mathrm{R} 22,22 / / 2 \mathrm{Cro}$ 18,21. Es éste un texto de hostilidad abierta de Yavé contra Ajab. El único, además de $\mathrm{Jr} 20,7$, en el que aparece $y k l$ no seguido de otro verbo y con un significado que, en contexto de oposición, está relacionado con la victoria. Por otra parte, se describe allí la «táctica» o «estratagema» del espíritu enviado por Yavé. Estos dos últimos términos son los que nos indican con más propiedad el ámbito semántico del PTH piel. La coloración concreta, de la cual depende la traducción, cambiará según las situaciones, pero siempre permanece este substrato nuclear configurante.

\section{A. RODRÍGUEZ MERINO}

